\title{
Primer registro de Nopachtus coagmentatus (Xenarthra, Glyptodontidae) para la Provincia de Catamarca, Argentina. Revisión del género Nopachtus
}

\author{
First record of Nopachtus coagmentatus (Xenarthra, \\ Glyptodontidae) for the Catamarca Province, Argentina. \\ Revision of the genus Nopachtus
}

M. Zamorano ${ }^{1}$, G.G. Moreno ${ }^{2}$, N.E. Vaccari ${ }^{3}$, C.M. Falcón ${ }^{4}$, A.M. Combina ${ }^{5}$, R.A. Bonini ${ }^{6}$

1 División Paleontología de Vertebrados, Museo de La Plata, Facultad de Ciencias Naturales y Museo, Universidad Nacional de La Plata. Paseo del Bosque s/n. Código postal 1900. La Plata. CONICET. Email: marzamorano@fcnym.unlp.edu.ar. ORCID ID: http://orcid.org/0000-0001-8538-256X

2 Dirección General de Minería - Universidad Nacional de La Rioja (UNLaR). Luis Vernet y Apóstol Felipe 5300. Código Postal 5300. La Rioja. Email: gabymor 89@hotmail.com. ORCID ID: http://orcid.org/0000-0003-1822-0426

3 CIPAL - CICTERRA (Facultad de Ciencias Exactas Físicas y Naturales Universidad Nacional de Córdoba - CONICET) Av. Vélez Sársfield 299. Código postal 5000, Córdoba. Email: evaccari@unc.edu.ar. ORCID ID: http://orcid. org/0000-0002-2965-1566

4 Instituto Superior de Correlación Geológica (INSUGEO). Universidad Nacional de Tucumán. Código Postal 4000. Email: camafa377@yahoo.com.ar. ORCID ID: http://orcid.org/0000-0002-2755-4519

5 Instituto de Geología y Recursos Naturales (INGEREN). Universidad Nacional de La Rioja. Dr. Luis Vernet 1000. Código Postal 5300. La Rioja, Argentina. Email: ana.maria.combina@gmail.com. ORCID ID: http://orcid.org/0000-0001-9125-1080

6 INCUAPA-CONICET, Facultad de Ciencias Sociales, Universidad Nacional del Centro de la Provincia de Buenos Aires, Olavarría, Argentina. Email: rbonini@fcnym.unlp.edu.ar. ORCID ID: http://orcid.org/0000-0002-1402-6530

\section{RESUMEN}

Nopachtus coagmentatus fue reconocido por Ameghino en 1888. El holotipo procede de la Formación Brochero (Montehermosense - Chapadmalalense [Plioceno temprano - Plioceno tardío]), de las cercanías de Villa Cura Brochero (Córdoba). Se reporta el primer hallazgo de esta especie para la Provincia de Catamarca. Se describe el material hallado, se detalla su procedencia y se presenta el perfil estratigráfico detallado correspondiente. El material mencionado, procedente de la Formación Salicas aflorante en el paraje de Suri Yaco, consiste en 14 osteodermos de la coraza dorsal que se atribuyen a $N$. coagmentatus, 12 completos y 2 fragmentados correspondientes a la región media y póstero-dorsal de la coraza dorsal. Estos osteodermos presentan una figura central rodeada por dos hileras de figuritas periféricas y figuritas que corresponderían a una tercera hilera, siempre incompleta. La primera hilera tiene entre 10 y 12 figuritas y la segunda entre 19 y 22; las figuritas son pentagonales o, mayoritariamente, hexagonales. A su vez, se revisa todo el material hasta ahora conocido para este género, que incluye dos especies. Para N. coagmentatus solamente había dos registros fehacientes, hasta esta comunicación, y varios dudosos, en tanto que para N. cabrerai Zamorano, Scillato-Yané, González Ruiz \& Zurita solo se dio a conocer el holotipo.

Palabras clave: Nopachtus coagmentatus; Formación Salicas; Catamarca; Gliptodóntidos.

Recibido el 15 de marzo de 2017 / Aceptado el 22 de junio de 2017 / Publicado online el 13 de septiembre de 2017

Citation / Cómo citar este artículo: Zamorano, M. et al. (2017). Primer registro de Nopachtus coagmentatus (Xenarthra, Glyptodontidae) para la Provincia de Catamarca, Argentina. Revisión del género Nopachtus. Estudios Geológicos 73(2): e067. http:// doi.org/10.3989/egeol.42809.449.

Copyright: () 2017 CSIC. This is an open-access article distributed under the terms of the Creative Commons Attribution-Non Commercial (by-nc) Spain 3.0 License. 


\begin{abstract}
Nopachtus coagmentatus was recognized by Ameghino in 1888. Holotype comes from Brochero Formation (Montehermosan - Chapadmalalan [early Pliocene - late Pliocene]), near to Villa Cura Brochero (Córdoba). First find of this species for the Catamarca Province is reported. Remains found are described, its provenance detailed and the corresponding stratigraphic log is presented. The mentioned material, came from Salicas Formation, cropping out in the paraje Suri Yaco, consists of 14 osteoderms of the dorsal region of the carapace attributed to $N$. coagmentatus, 12 complete and 2 fragmented corresponding to the media and posterodorsal region of the carapace. These osteoderms present a central figure surrounded by two row of peripheral figures, and figures that would correspond to a third row, always incomplete. The first row has between 10 and 12 figures and the second between 19 and 22; the figures are pentagonal or, mainly, hexagonal. In turn, all specimens known today for this genus, that included two species, is revised. Until this communication, only two reliable records, and several doubtful were recognized for N. coagmentatus; while for N. cabrerai Zamorano, Scillato-Yané, Gonzalez Ruiz \& Zurita only the holotype was given to known.
\end{abstract}

Keywords: Nopachtus coagmentatus; Salicas Formation; Catamarca; Glyptodontids.

\section{Introducción}

Los gliptodóntidos integran el grupo de Cingulata (Xenarthra) cuyos representantes alcanzan mayor tamaño, a veces gigantesco (Scillato-Yané \& Carlini, 1998; Fariña, 2001; Soibelzon et al., 2012; Zamorano et al., 2014). La familia Glyptodontidae se registra desde el Eoceno medio hasta el Holoceno temprano (Scillato-Yané, 1976; Fernicola, 2008; Soibelzon et al., 2012; Zamorano, 2013). Hoffstetter (1958) realiza una clasificación, de alta aceptación en el ámbito científico, que divide a la familia en cinco subfamilias (Glyptatelinae, Propalaehoplophorinae, Doedicurinae, Glyptodontinae y Hoplophorinae).

La subfamilia "Hoplophorinae" se registra desde el Mioceno medio hasta el Holoceno temprano, su característica más notoria es la precoz adquisición de un tubo caudal (Zurita, 2007; Zamorano, 2012a). No obstante, esta subfamilia no es considerada un grupo natural (Fariña, 1993; Fernicola, 2008; Porpino et al., 2010; Fernicola \& Porpino, 2012).

Nopachtus Ameghino tradicionalmente fue considerado en una de las tribus de los "Hoplophorinae", junto con los géneros Propanochthus Castellanos y Panochthus Burmeister, la Tribu "Panochthini", la cual corresponde a un agrupamiento no natural (Zamorano, 2012a; Zamorano \& Brandoni, 2013; Zamorano et al., 2014).

Ameghino (1888a) establece el género Nopachtus para incluir a $N$. coagmentatus Ameghino, especie reconocida sobre la base de un gran trozo de coraza dorsal y segmentos del tubo caudal. Castellanos (1925) añade a este género la especie $N$. trouessarti (Moreno, 1888), reconocida basándose en la mitad de la coraza dorsal. Zamorano et al. (2011) transfieren $N$. trouessarti a Phlyctaenopyga Cabrera, 1944 y reconocen N. cabrerai Zamorano, Scillato-Yané, González Ruiz \& Zurita, 2011. De esta manera, Nopachtus queda constituido por dos especies: $N$. coagmentatus y N. cabrerai (ver Zamorano, 2012a, 2012b; Zamorano et al., 2015; Zamorano et al., 2016).

El holotipo de $N$. coagmentatus se registra para la Formación Brochero (Montehermosense Chapadmalalense [Plioceno temprano - Plioceno tardío] [ver Cruz, 2013]) de las Sierras de Córdoba, en las cercanías de Villa Cura Brochero. A su vez, el holotipo de $N$. cabrerai se registra en la Formación Monte Hermoso (Montehermosense, Plioceno temprano) de la localidad Farola Monte Hermoso (Buenos Aires) (Zamorano et al., 2011; Zamorano, 2012a, 2012b; Tomassini et al., 2013; Zamorano et al., 2015). Además del holotipo, hay otra cita fehaciente para esta especie basada en osteodermos de la coraza dorsal, procedentes de la Formación El Polvorín (Chapadmalalense superior, Plioceno tardío) de las Sierras Bayas de Olavarría (Buenos Aires) (Zamorano et al., 2015); también existen algunas menciones dudosas (Ameghino 1888b; Castellanos, 1937, 1942a, 1942b, 1944; Rusconi, 1948, 1949; Cruz, 2011; Zamorano et al., 2011, 2016; Chimento, 2012; Zamorano, 2012a).

En esta contribución se presenta y describe el primer registro de Nopachtus coagmentatus para la provincia de Catamarca. Se revisa el género Nopachtus, brindando las diagnosis de los taxones incluidos. También se discute sobre la posición sistemática de los materiales alguna vez atribuidos a Nopachtus. Asimismo, se detalla el perfil estratigráfico del lugar de hallazgo y se realiza un aporte a la 
discusión de la edad de la Formación Salicas. A su vez, se elabora un mapa geológico de la zona.

\section{Procedencia Geográfica y Estratigráfica}

El material estudiado fue hallado durante los trabajos de relevamiento de campo de la tesis de grado de Moreno (2015) en el paraje Suri Yaco, $21 \mathrm{~km}$ al suroeste de la localidad de Pomán, Departamento Pomán, Provincia de Catamarca y a $18 \mathrm{Km}$ al noreste de las Termas de Santa Teresita, en el Departamento Aimogasta, La Rioja 28 $35^{\prime} 44.59$ ' S y $66^{\circ} 32 ' 58.66^{\prime}$ W (Fig. 1, 2). En esta localidad afloran las Formaciones Salicas (niveles superiores) y Las Cumbres (Sosic, 1973). Sosic (1973), señala que en esta localidad afloran las Formaciones Salicas y Las Cumbres, sin embargo en el sitio del descubrimiento solo afloran los niveles superiores de la Formación Salicas, la cual está cubierta discordantemente por la Formación Las Cumbres. El contacto entre estas formaciones y el basamento no fue reconocido en el afloramiento, sin embargo, Sosic (1973) propone que este seria de origen tectónico. La columna estratigráfica presenta la sucesión sedimentaria y el nivel del hallazgo de los restos fósiles reportados en este trabajo (Fig. 3).

La secuencia sedimentaria correspondiente a la Formación Salicas (Mioceno superior - Plioceno inferior?), donde se encontró el fósil descripto en este trabajo, está constituida por intercalaciones de facies arenosas y loéssicas que alcanzan un espesor medido en el afloramiento de hasta 4,5 m. Estas últimas corresponderían a depósitos de ríos entrelazados y de llanuras de inundación, con alternancias periódicas de inundación y desecación manifestadas por la presencia de calcretes y grietas de desecación. Estos depósitos pudieron formarse en un clima semiárido dominante como el que rige actualmente. Por encima de la Formación Salicas y en relación de discordancia angular, se dispone la Formación Las Cumbres (Plioceno/Pleistoceno?).

\section{Materiales y Métodos}

El material estudiado se compone de un conjunto de 14 osteodermos de la coraza dorsal, se encuentra depositado en el Museo de Ciencias Antropológicas y Naturales de la Universidad Nacional de La Rioja

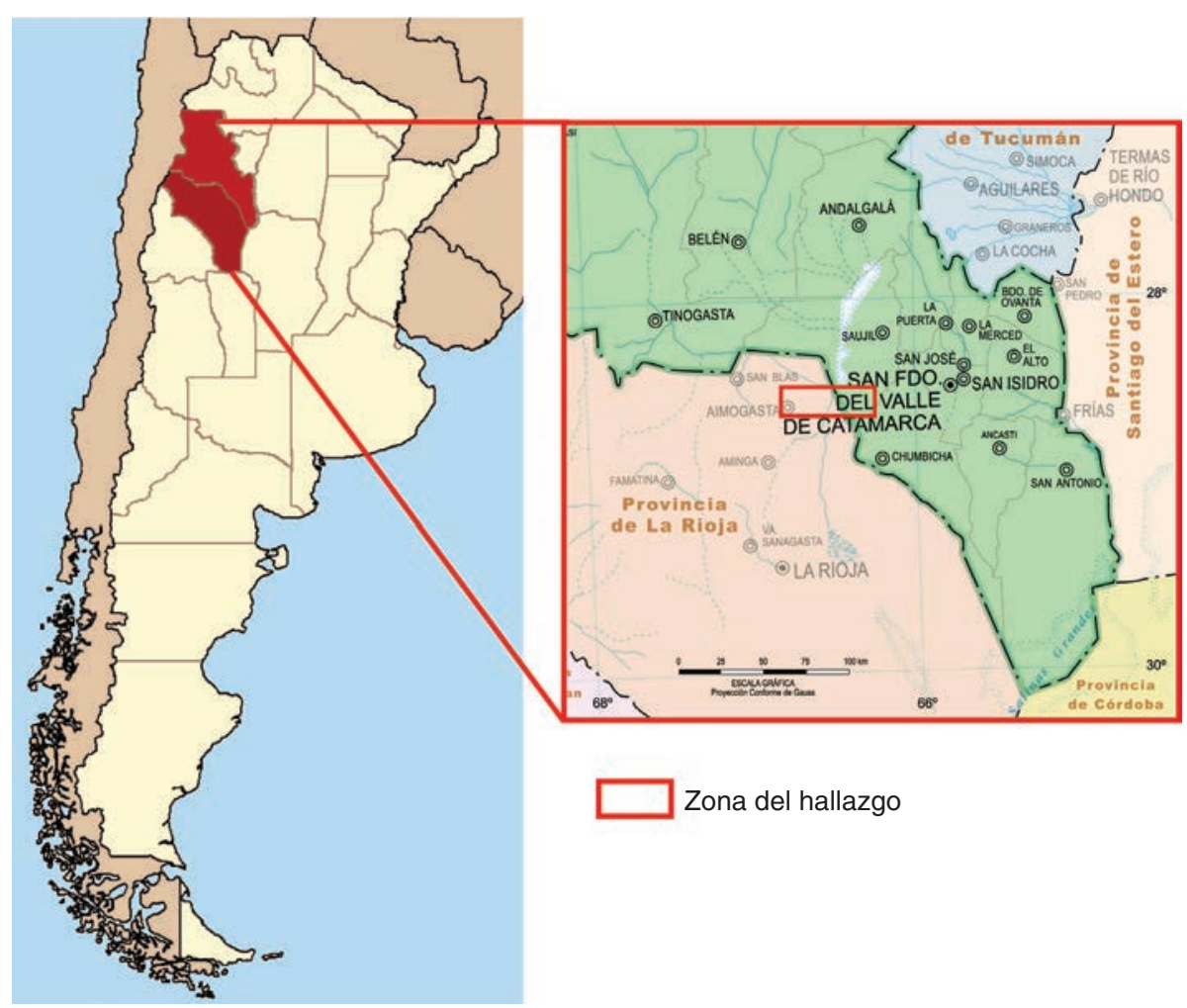

Fig. 1.-Ubicación geográfica del afloramiento de la Formación Salicas. 


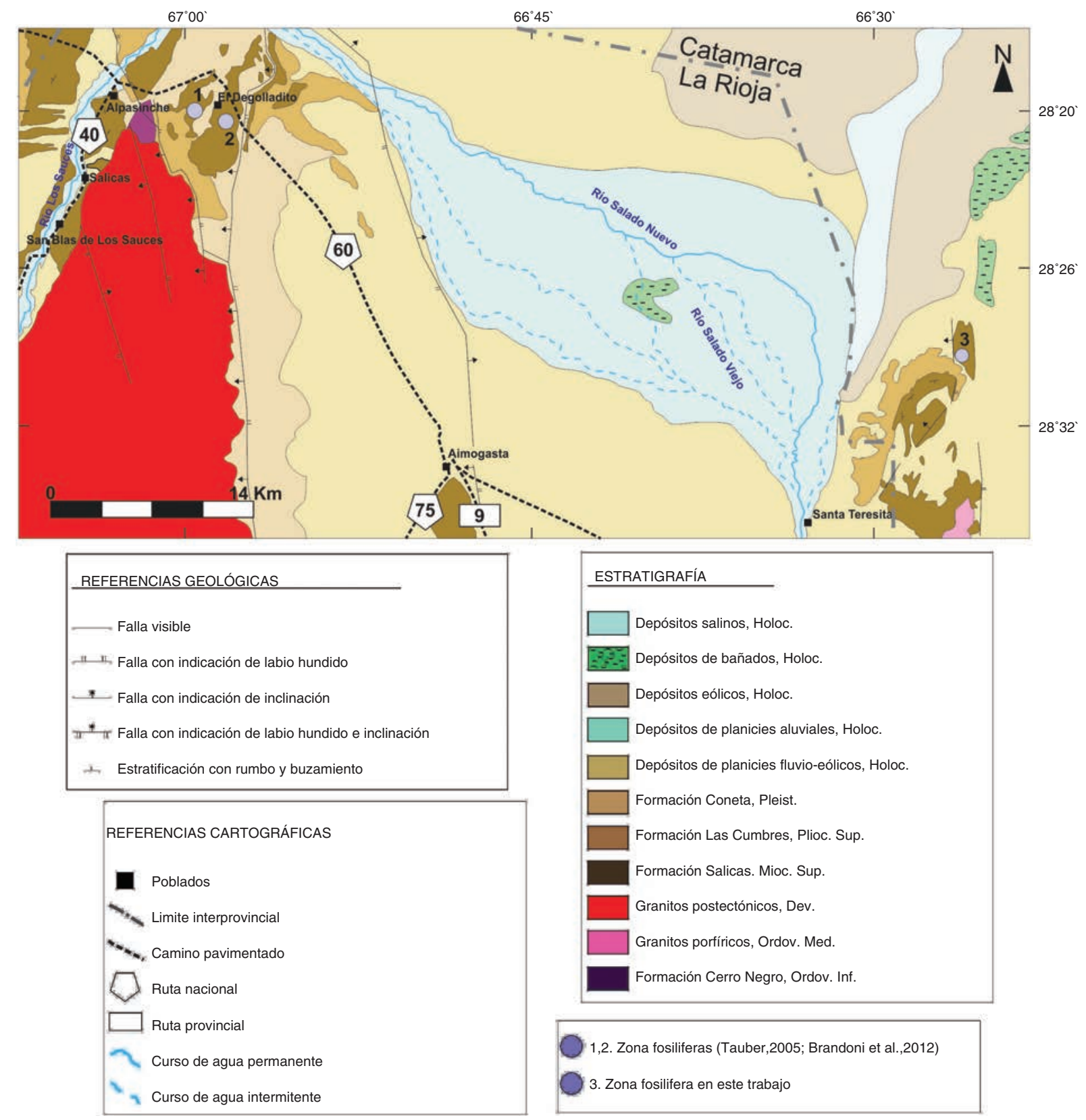

Fig. 2.-Mapa geológico del área de hallazgo.

y ha sido inventariado con el acrónimo PULR-V 112. El mismo ha sido comparado con el material tipo de Nopachtus coagmentatus con procedencia geográfica y estratigráfica conocida, MLP 122 (holotipo) y Xen-45. Para realizar las descripciones y comparaciones se utilizó la terminología anatómica de Zamorano (2012a) y Porpino et al. (2014, fig. 2D). Para la sistemática se siguió a Zamorano \& Brandoni (2013) (ver Zamorano et al., 2015). La nomenclatura estratigráfica se basó en Sosic (1973).
Abreviaturas utilizadas: LIL-PV, Paleontología de Vertebrados del Instituto Miguel Lillo (San Miguel de Tucumán, Tucumán); MACN A, Museo Argentino de Ciencias Naturales "Bernardino Rivadavia", Colección Ameghino (Ciudad Autónoma de Buenos Aires); MLP, División de Paleontología de Vertebrados, Facultad de Ciencias Naturales y Museo de La Plata (La Plata, Buenos Aires); MUFyCA, Museo Universitario Florentino y Carlos Ameghino 


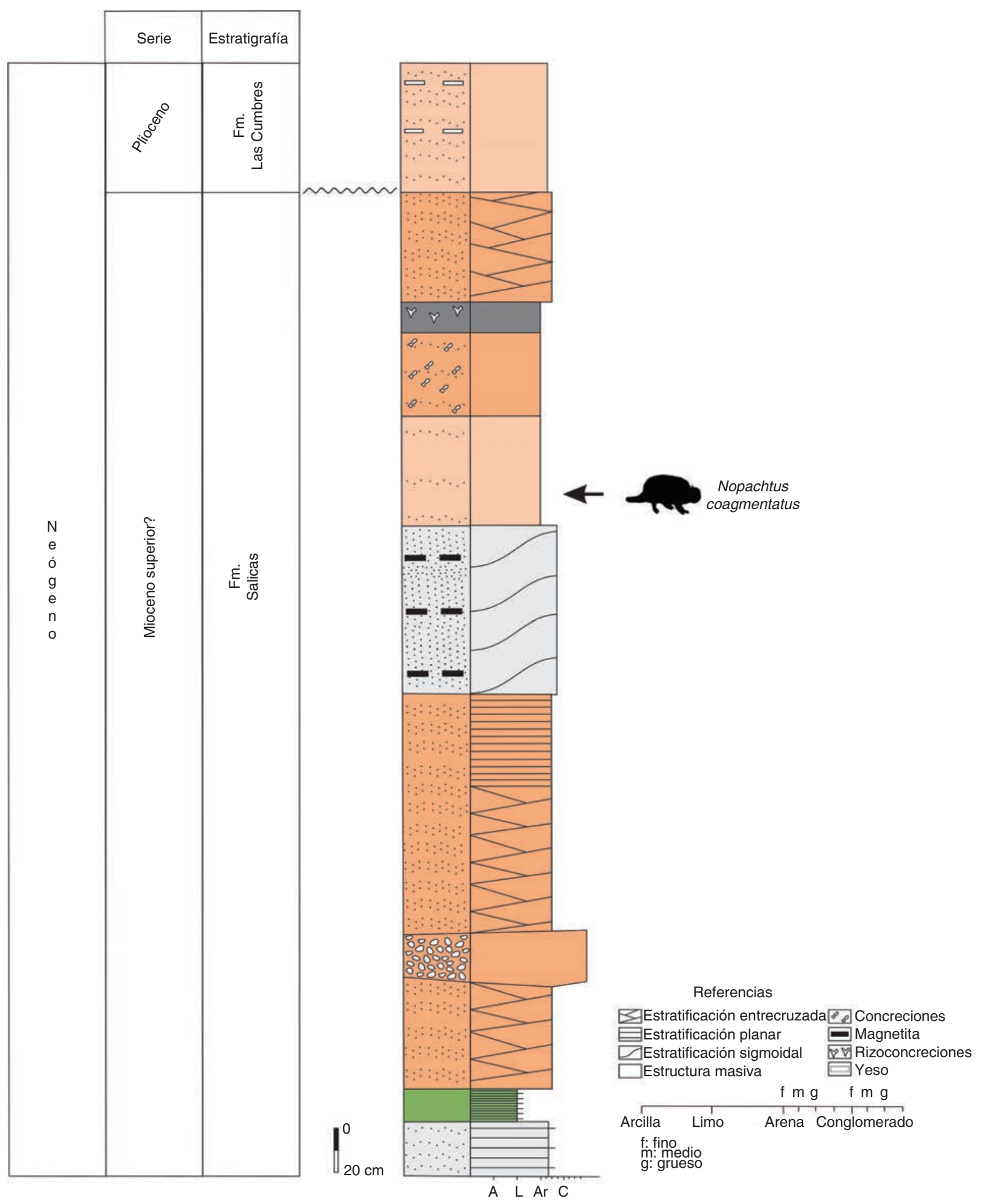

Fig. 3.-Perfil estratigráfico del sector estudiado. 
(Rosario, Santa Fe); Xen, Colección Cementos Avellaneda (Olavarría, Buenos Aires).

\section{Sistemática Paleontológica}

Magnorden XENARTHRA Cope, 1889

Orden CINGULATA Illiger, 1811

Suborden GLYPTODONTIA Ameghino, 1889

Superfamilia GLYPTODONTOIDEA Gray, 1869

Familia GLYPTODONTIDAE Gray, 1869

Género Nopachtus Ameghino, 1888a

1988. Panochthus Moreno, p. 8.

1895. Panochthus Lydekker, p. 28.

1925. Nopachthus Castellanos, 1925 y publicaciones posteriores del autor, emmendatio illegitima pro Nopachtus Ameghino, 1888a (ver Mones, 1986: 237; ICZN, 1999: Art. 33).

Diagnosis (enmendada de Zamorano, 2012a): Gliptodóntidos de talla mediana a mediano-grande, algo mayor que Phlyctaenopyga pero menor que Panochthus. Osteodermos de la coraza dorsal con la figura central rodeada generalmente por dos hileras de figuritas periféricas (la segunda hilera no siempre completa). Figuritas periféricas de la primera hilera que rodea la figura central en número de 8 a 12; en algunos osteodermos donde la segunda hilera es completa se cuentan hasta 23 figuritas. Las figuritas son pentagonales o, mayoritariamente, hexagonales. La figura central es más elevada que las figuritas en la región posterior, y plana, hasta levemente deprimidas, en la anterior.

Especies referidas: $N$. coagmentatus y $N$. cabrerai (ver más abajo las respectivas diagnosis).

Nopachtus coagmentatus Ameghino, 1888a

1988. Panochthus brocherii Moreno (nomen nudum), p. 9. 1895. Panochthus bullifer Lydekker, 1895 p. 31-33, pl. XVII, fig. 1, 2.

Diagnosis (enmendada de Zamorano, 2012a): Osteodermos de la coraza dorsal con la figura central rodeada por una primera hilera de 8 a 12 figuritas periféricas, a su vez rodeada, solamente en algunos osteodermos, por una segunda hilera de hasta 21 . Solamente en el tercio posterior de la coraza las figuras centrales de los osteodermos son más elevadas que las periféricas. Dichas figuras centrales son mucho menos elevadas que en $N$. cabrerai. Tubo caudal cónico-cilíndrico, con grandes figuras abultadas.

Material examinado: PULR-V 112, 14 osteodermos, 12 completos y 2 incompletos, correspondientes a la región medio y postero-dorsal de la coraza dorsal (Fig. 4).

Procedencia estratigráfica y geográfica del material examinado: Facies loéssica de la Formación Salicas (Mioceno superior - Plioceno inferior?) (Fig. 3) del paraje de Suri Yaco (Catamarca) (Fig. 1, 2).

Descripción: Los osteodermos presentan una figura central rodeada por dos hileras de figuritas periféricas. La primera hilera tiene entre 10 y 12 figuritas periféricas y la segunda entre 19 y 22 , la forma que pueden presentar las figuritas son pentagonales $\mathrm{o}$, mayoritariamente, hexagonales, como en $N$. cabrerai. En todos los osteodermos se puede apreciar la presencia de figuritas, que corresponderían a una tercera hilera, la cual siempre está incompleta. La primera hilera de figuritas periféricas presenta una menor cantidad respecto de Stromaphorus compressidens (Moreno \& Mercerat) (12 a 14, ver Cabrera, 1944: 34) y que en la región medio y postero-dorsal de Phlyctaenopyga ameghini Ameghino, (14 a 18, ver Zamorano et al., 2011, fig. 2), sin embargo, hay mayor cantidad de figuritas que en Plohophorus figuratus Ameghino, (6 a 10), y, aproximadamente la misma cantidad que en Pseudohoplophorus absolutus Perea ("siempre en número total de 11 o más y muy pequeñas en relación a la figura central" Perea, 2005: 177). A su vez, la cantidad de figuritas periféricas de la segunda hilera también permite

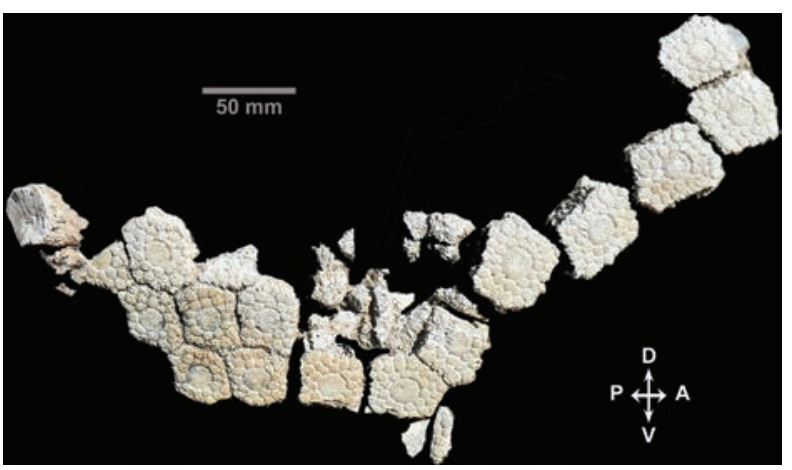

Fig. 4.-Osteodermos de la coraza dorsal (PULR-V 112-51) atribuidos a Nopachtus coagmentatus, ubicados en la posición que tienen en la coraza. A, anterior; D, dorsal; P, posterior; V, ventral. 
diferenciarlo de las especies recientemente mencionadas, de Plohophorus figuratus (16 a 17, según Ameghino, 1889), Pseudohoplophorus absolutus (2 figuritas, solo en algunas placas de la zona lateral, ver Perea, 2005) y de Stromaphorus compressidens (entre 18 y 21, ver Cabrera, 1944, fig. 18) que tienen menos figuritas, y de Phlyctaenopyga ameghini (de 23 a 27, ver Zamorano et al., 2011), que posee más figuritas. Las figuras centrales son mayoritariamente planas, y la de algunos osteodermos, levemente deprimidas, a diferencia de $N$. cabrerai, que en la misma región, presenta una figura central elevada a francamente convexa, con aspecto de ampollas.

\section{Nopachtus cabrerai Zamorano, Scillato-Yané, González Ruiz \& Zurita, 2011}

1942a. Nopachtus trouessarti Castellanos p. 459464, fig. 188, 189.

Diagnosis (enmendada de Zamorano, 2012a): Osteodermos de la coraza dorsal con la figura central rodeada por una primera hilera de hasta 10 a 12 figuritas periféricas; a su vez rodeada, solamente en algunos osteodermos, por una segunda hilera de hasta 21 figuritas. Las figuras centrales en la región anterior son planas En la mitad posterior estas figuras son mucho más elevadas que las figuritas periféricas. A su vez, en el tercio más posterior dichas figuras centrales son mucho más elevadas que en $N$. coagmentatus, en forma de verdaderas ampollas.

\section{Revisión de los materiales atribuidos a las especies del género Nopachtus}

El holotipo (MLP 16-122), gran porción de coraza dorsal y un fragmento (originalmente tres, ver Zamorano, 2012a) de tubo caudal, procedente de los valles de las Sierras de Córdoba (Ameghino, 1888a), y Xen-45, osteodermos de la coraza dorsal, que provienen de Olavarría (Zamorano et al., 2015) son, hasta ahora, los únicos registros fehacientes de Nopachtus coagmentatus (Fig. 5). A estos

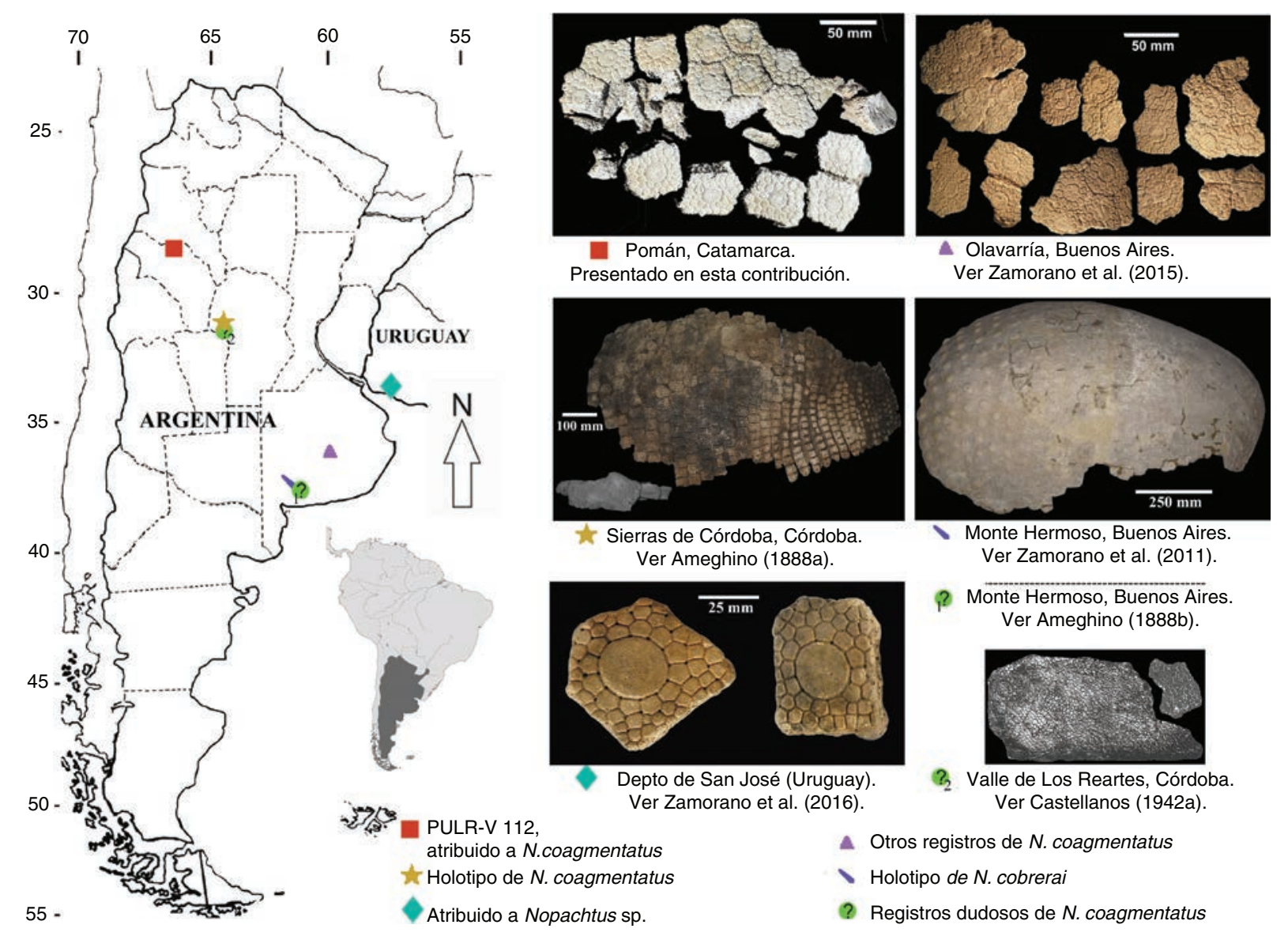

Fig. 5.-Distribución geográfica de los materiales atribuidos al género Nopachtus. 
materiales se le suman algunos registros dudosos, Ameghino (1888b: 479-480) asigna a $N$. coagmentatus "...un trozo considerable de coraza", de Monte Hermoso, pero sin justificar dicha asignación, pues no lo describe ni figura, tampoco indica dónde está depositado; sumado esto a la circunstancia de que Ameghino obviamente desconocía a $N$. cabrerai, por lo que la mención de $N$. coagmentatus en Monte Hermoso por parte de Ameghino resulta sumamente dudosa (ver Zamorano et al., 2015) (Fig. 5). Por su parte, Castellanos (1942a: 433-434, figs. 171 y 172) da cuenta de un fragmento de coraza dorsal procedente del "Brocherense" del Valle de Los Reartes (Córdoba), sin indicar ni el repositorio ni el número del ejemplar (Fig. 5). Además, Castellanos (1937, 1942a, 1942b, 1944) atribuye materiales muy dudosos a esta especie, algunos de los cuales son reasignados por Cruz (2011), MUFyCA 1017 (osteodermos aislados y fragmentos de coraza dorsal) a Phlyctaenopyga sp., MUFyCA 370 (osteodermos de la coraza dorsal) a Propanochthus bullifer, y MUFyCA 396 (fragmento de coraza dorsal) a Plohophorus sp. (ver Cruz, 2011, 2013). Rusconi $(1948,1949)$ figura y da las medidas de dos osteodermos $\left(\mathrm{N}^{\circ} 1159\right.$ y N $\mathrm{N}^{\mathrm{o}} 1322$, de su colección) procedentes del "Puelchense" (sensu Rusconi, 1948; 1949) de Villa Ballester (Buenos Aires) que atribuye a $N$. coagmentatus, los cuales menciona (1948: 89-90) y figura (1849: lám. XI, figs. 6 y 7), respectivamente; sin indicar dónde están depositados. Se considera que estos restos son asignables a Propanochthus bullifer, coincidiendo con Zamorano (2012a) y

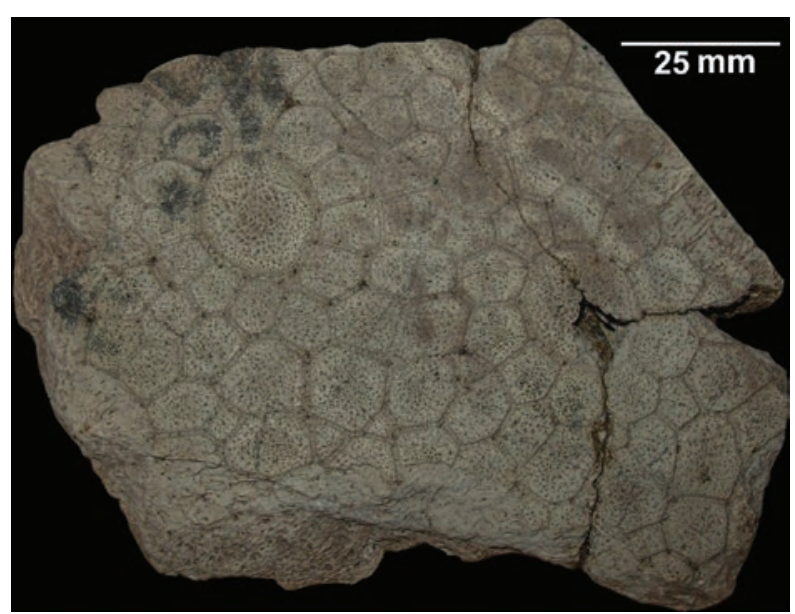

Fig. 6.-MACN A 1208/09.Fragmento de la coraza dorsal atribuido a Nopachtus sp. difiriendo con Chimento (2012), este último reasigna el N. ${ }^{\circ} 1322$ (LIL-PV 768) a Panochthus sp. Además, Rusconi (1948: 90) menciona otro osteodermo, manifestando: "Posiblemente, la (placa) $n^{o}$ 1034 corresponda a este género (Nopachtus), pero su escultura se halla muy destruida" (sic), no lo figura, ni lo describe, no existen elementos de juicio suficientes como para formarse una opinión.

En Zamorano et al. (2011) y en Zamorano (2012a) se menciona, en el hipodigma de la "Sistemática y Paleontológica", el material MACN A 1208/09 correspondiente a un fragmento de coraza dorsal (Fig. 6). En el presente trabajo, este último material, es reasignado como Nopachtus sp., ya que es muy riesgoso realizar una determinación específica al no poderse corroborar a que región de la coraza dorsal corresponde el material. Además, cabe aclarar que el material no tiene datos de procedencia.

Por último Zamorano et al., 2016 atribuyen a Nopachtus sp. dos osteodermos de la coraza dorsal provenientes o bien de la Formación Camacho (Mioceno Tardío) o de la Formación Raigón(Plioceno Tardío - Pleistoceno Medio) del Departamento de San José (Uruguay) (Fig. 5).

\section{Discusión}

\section{Consideraciones sistemáticas}

El material estudiado presenta más osteodermos con figuritas periféricas correspondientes a la tercera hilera, que en el holotipo y en el Xen-45 (ver Zamorano et al., 2015). El aspecto de este material se asemeja al de la figura de la coraza del holotipo que se ve en Lydekker (1895, pl. XVII) y en Castellanos (1942a, fig. 170), en estas figuras la coraza del holotipo se encuentra más completa respecto de cómo se halla en la actualidad (Zamorano, 2012a). En estas últimas se observa la región a la cual corresponderían los osteodermos del material de Catamarca; en esta región, medio postero-dorsal, se verifica un aumento en las mencionadas figuritas de la tercera hilera.

\section{Consideraciones sobre la edad de la Formación Salicas}

Tauber (2005) estudió la Formación Salicas y recogió vertebrados fósiles en la zona de La Cortadera y El Degolladito, cerca del pueblo de Alpasinche. 
Los taxones registrados fueron: Macrochorobates scalabrinii (Moreno \& Mercerat), Proeuphractus limpidus (Ameghino), Chaetophractus sp., Eosclerocalyptus planus (Rovereto), Neophanomys biplicatus (Rovereto), Lagostomus (Lagostomopsis) cf. L. pretrichodactyla, Orthomyctera andina Kraglievich, Potamarchus sp., Protypotherium sp., Pseudotypotherium sp., Hemihegetotherium cf. $H$. torresi y cf. Tremacyllus sp. Este autor sugiere para esta asociación una edad Huayqueriense, y una menos probable Montehermosense. Por otra parte, Brandoni et al. (2012), en el área de El Degolladito, encontró la siguiente asociación: Macrochorobate sp., Chasicotatus sp., Hoplophorini indet., Paedotherium minor Cabrera, cf. Pseudotypotherium Ameghino, Neobrachytherium sp., Orthomyctera sp., cf. Cardiomys Ameghino, Lagostomus (Lagostomopsis) sp. y Octodontidae indet. Basándose en la presencia de estos géneros, Brandoni et al. (2012) mencionan que la Formación Salicas tiene una mayor afinidad con la Formación Cerro Azul de la provincia de La Pampa, que con el Miembro El Jarillal de la Formación Chiquimil y la Formación Andalhuala de la provincia de Catamarca, y consideran que la fauna de la Formación Salicas pertenece al Mioceno tardío sin precisar más hasta que nuevos descubrimientos de fósiles y dataciones radio-isotópicas permitan una mejor calibración de la misma.

El hallazgo de $N$. coagmentatus reportado aquí, aporta nuevos elementos a la discusión de la edad de la Formación Salicas. Esta especie fue reconocida por Ameghino en 1888, en las cercanías de Villa Cura Brochero; en la Formación Brochero [Montehermosense - Chapadmalalense (Plioceno temprano - Plioceno tardío)] (Cruz et al., 2013). Además del holotipo, hay otra cita fehaciente para esta especie, que consiste en la presencia de osteodermos de la coraza dorsal, procedentes de la Formación El Polvorín (Chapadmalalense superior, [Plioceno tardío]) de las Sierras Bayas, Olavarría (Buenos Aires) (Zamorano et al., 2015). $\mathrm{Si}$ bien es aún prematuro afirmar la edad precisa de este hallazgo, el mismo podría confirmar la edad miocena tardía sugerida por Brandoni et al. (2012) e incluso evidenciar la presencia de niveles algo más jóvenes de la Formación Salicas que podrían alcanzar el Plioceno en la localidad de Suri Yaco. Una búsqueda intensiva de fósiles en esta localidad permitirá precisar la edad de estos niveles.

\section{Conclusiones}

El género Nopachtus incluye dos especies, $N$. coagmentatus y $N$. cabrerai. El material aquí presentado es asignado a $N$. coagmentatus y atribuido a la región medio y postero-dorsal de la coraza dorsal por las siguientes características: los osteodermos presentan una figura central rodeada por dos hileras de figuritas periféricas; la primera hilera incluye entre 10 y 12 figuritas periféricas pentagonales o hexagonales; una segunda hilera que tiene entre 19 y 22 figuritas, también hay figuritas que corresponderían a una tercera hilera, siempre incompleta. $N$. coagmentatus, hasta la presente contribución, se registraba fehacientemente para la Formación Brochero (Montehermosense Chapadmalalense [Plioceno temprano - Plioceno tardío]) de las Sierras de Córdoba y para el Chapadmalalense superior de Sierras Bayas de Olavarría (Buenos Aires). A partir de este nuevo hallazgo se amplía la distribución estratigráfica y geográfica al Mioceno superior - Plioceno inferior de Catamarca y por primera vez se describe material de Nopachtus coagmentatus hallado en la región noroeste del país.

\section{AGRADECIMIENTOS}

A la Universidad de La Rioja y al proyecto 27/A520 "Estudios Hidrogeotermales en el sector centro-norte de la provincia de La Rioja y posibles aplicaciones de sus aguas termales e baja entalpía", por brindar los medios para realizar la campaña. A Gustavo J. Scillato-Yané, por responder consultas sobre el manuscrito. A los revisores, Daniel Perea y Ángel R. Miño Boilini, por enriquecer el trabajo.

\section{Referencias}

Ameghino, F. (1888a). Rápidas diagnosis de algunos mamíferos fósiles nuevos de la República Argentina. Pablo E. Coni e Hijos, Buenos Aires. 17 pp.

Ameghino, F. (1888b). Lista de las especies de mamíferos fósiles del mioceno superior de Monte Hermoso, hasta ahora conocidas. Pablo E. Coni e Hijos, Buenos Aires. 21 pp.

Ameghino, F. (1889). Contribución al conocimiento de los mamíferos fósiles de la República Argentina. 
Actas Academia Nacional de Ciencias de Córdoba 6: 32-1028 y Atlas de 98 láminas.

Brandoni, D.; Schmidt, G.I.; Candela, A.M.; Noriega, J.I.; Brunetto, E. \& Fiorelli, L.E. (2012). Mamíferos de la formación Salicas (Mioceno tardío), Provincia de La Rioja, Noroeste de Argentina: paleobiogeografía, edad y paleoambiente. Ameghiniana, 49 (3): 375-387. https://doi.org/10.5710/AMGH.v49i3(467)

Cabrera, A. (1944). Los Gliptodontoideos del Araucaniano de Catamarca. Revista del Museo de La Plata (N. Serie), Paleontología, 3: 5-76.

Castellanos, A. (1925). Breves notas sobre evolución de la coraza y del extremo caudal en los Loricata (=Hicanodonta). Revista de "El Circulo", número extraordinario. Rosario de Santa Fe, 93-96.

Castellanos, A. (1937). Los sedimentos del Pampeano inferior y del Araucano en el Valle de Los Reartes (Sierra de Córdoba). Publicaciones de la Facultad de Ciencias Matemáticas, Físico-Químicas y Naturales aplicadas a la Industria, (serie Técnico-Científica) 6, Universidad Nacional del Litoral, Rosario, 110 pp.

Castellanos, A. (1942a). A propósito de los géneros Plohophorus, Nopachthus y Panochthus (3a. parte). Publicación del Instituto de Fisiografía y Geología de la Universidad Nacional del Litoral, 11: 413-592.

Castellanos, A. (1942b). Los sedimentos prepampeanos del Valle del Nono (Sierra de Córdoba) Argentina. Publicaciones del Instituto de Fisiografía y Geología de la Universidad Nacional del Litoral, 13: 5-62.

Castellanos, A. (1944). Paleontología estratigráfica de los sedimentos neógenos de la provincia de Córdoba. Publicaciones del Instituto de Fisiografía y Geología de la Universidad Nacional del Litoral, 23: 5-47.

Chimento, N. (2012). Revisión de algunos Xenarthra (Mammalia) del «Puelchense» de la provincia de Buenos Aires, Argentina. Historia Natural, Tercera serie, 2 (1): 5-40.

Cruz, L. (2011). La megafauna del Pleistoceno - Holoceno temprano de la Provincia de Córdoba y su comparación con la de Buenos Aires. Sistemática, Bioestratigrafía y Dinámica Faunística. Tesis doctoral, Universidad Nacional de la Plata, 226 pp.

Cruz, L. (2013). Biostratigraphy and geochronology of the late Cenozoic of Córdoba Province (central Argentina) Journal of South American Earth Sciences, 42: 250-259. https://doi.org/10.1016/j. jsames.2012.06.015

Fariña, R. (1993). Análisis filogenético de Glyptodontia. Ameghiniana 30 (3): 329-330.

Fariña, R. (2001). Física y Matemáticas para reconstruir la vida en el pasado. Actas de Fisiología, 6:45-70.

Fernicola, J. (2008). Nuevos aportes para la sistemática de los Glyptodontia Ameghino 1889 (Mammalia, Xenarthra, Cingulata). Ameghinana 45 (3): 553-574.

Fernicola, J. \& Porpino K. (2012). Exoskeleton and Systematics: A Historical Problem in the Classification of Glyptodonts. Journal of Mammalian
Evolution 19 (1): 1-13. https://doi.org/10.1007/ s10914-012-9186-1

Hoffstetter, R. (1958). Xenarthra. In: Traité de Paléontologie (Piveteau, J., Ed.), Paris, 6 (2): 535-636.

Lydekker, R. (1895). Contributions to a knowledge of the Fossil Vertebrates of Argentina. 2. The Extinct Edentates of Argentina. Anales del Museo de La Plata (Paleontología Argentina), 3: 1-118.

Mones, A. (1986). Palaeovertebrata Sudamericana. Catálogo Sistemático de los Vertebrados fósiles de América del Sur. Parte I. Lista Preliminar y Bibliográfica. Courier Forschungsinstitut Senckenberg, 82: $1-625$.

Moreno, F. (1888). Informe preliminar de los progresos del Museo La Plata durante el primer semestre de 1888, presentado al señor ministro de Obras Públicas de la provincia de Buenos Aires. Boletín del Museo La Plata, 35 pp.

Moreno, G. (2015). Estudios Hidrogeotermales en el Área de Santa Teresita, La Rioja, Argentina. Tesis de Grado de la carrera de Geología, Departamento Académico de Ciencias y Tecnologías Aplicadas a la Producción, al Ambiente y al Urbanismo. Universidad Nacional de La Rioja, 164 pp.

Perea, D. (2005). Pseudoplohophorus absoluts n. sp. (Xenarthra, Glyptodontidae), variabilidad en Sclerocalyptinae y redefinición de una biozona del Mioceno Superior de Uruguay. Ameghiniana 42: 175-190.

Porpino, K.; Fernicola, J.C. \& Bergqvist L.P. (2010). Revisting the intertropical brazilian species Hoplophorus euphractus (Cingulata, Glyptodontoidea) and the phhylogenetic affinities of Hoplophorus. Journal of Vertebrate Paleontology 30 (3): 911-927. https:// doi.org/10.1080/02724631003765735

Porpino K.; Fernicola, J.C.; Cruz, L.E.\& Bergqvist, L.P. (2014) . The intertropical Brazilian species of Panochthus (Xenarthra, Cingulata, Glyptodontoidea): a reappraisal of their taxonomy and phylogenetic affinities. Journal of Vertebrate Palaeontology 34 (5): 1165-1179. https://doi.org/10.1080/0272463 4.2014 .863203

Rusconi, C. (1948). El Puelchense de Buenos Aires y su fauna (Plioceno medio). (Primera parte). Publicaciones del Instituto de Fisiografía y Geología de la Universidad Nacional del Litoral, 33: 1-99.

Rusconi, C. (1949). El Puelchense de Buenos Aires y su fauna (Plioceno medio). (Segunda parte). Publicaciones del Instituto de Fisiografía y Geología de la Universidad Nacional del Litoral, 36: 100-242.

Scillato-Yané, G.J. (1976). Sobre un Dasypodidae (Mammalia, Xenarthra) de Edad Riochiquense (Paleoceno Superior) de Itaboraí, Brasil. Anales de la Academia Brasilera de Ciências 48 (3): 529-530.

Scillato-Yané, G.J. \& Carlini, A. (1998). Un Gigantesco Gliptodonte en los Alrededores de la Ciudad de La Plata. Revista Museo,11 (2): 45-48. 
Sosic, M. (1973). Descripción geológica de la hoja 14e Salar de Pipanaco. Dirección Nacional de Geología y Minería, Boletín, 137: 1-47.

Soibelzon, L.H.; Zamorano, M.; Scillato-Yané, G.J.; Piazza, D.; Rodríguez, S.; Soibelzon, E.; Tonni, E.P.; San Cristóbal, J. \& Beilinson, E. (2012). Un glyptodontidae de gran tamaño en el Holoceno temprano de la región Pampeana. Revista Brasileira de Paleontologia, 15:113-122. https://doi.org/10.4072/rbp.2012.1.09

Tauber, A. (2005). Mamíferos fósiles y edad de la Formación Salicas (Mioceno tardío) de la Sierra de Velasco, La Rioja, Argentina. Ameghiniana, 42 (2): 443-460.

Tomassini, R.; Montalvo, C.; Deschamps, C. \& Manera, T. (2013). Biostratigraphy and biochronology of the Monte Hermoso Formation (earlyPliocene) at its type locality, Buenos Aires Province, Argentina. Journal of South American Earth Sciences, 48: 31-42. https://doi.org/10.1016/j.jsames.2013.08.002

Zamorano, M. (2012a). Los Panochthini (Xenarthra, Glyptodontidae): Sistemática y evolución. Tesis Doctoral, Universidad Nacional de La Plata, La Plata, Argentina, 269 pp.

Zamorano, M. (2012b). Los Panochthini (Xenarthra, Glyptodontidae): Sistemática y evolución. Mastozoología Neotropical, 19: 382-384.

Zamorano M. (2013). Diagnosis y nueva descripción de Propanochthus bullifer (Burmeister) (Xenarthra, Glyptodontidae). Consideraciones bioestratigráficas y cronológicas de su procedencia. Spanish Journal of Palaeontology, 28 (2): 285-294.

Zamorano, M.; Scillato-Yané G.; Gonzalez-Ruiz, L.R. \& Zurita, A.E, (2011). Revisión de los géneros
Nopachtus Ameghino y Phlyctaenopyga Cabrera (Xenarthra, Glyptodontidae, Hoplophorinae) del Mioceno tardío y Plioceno de Argentina. Revista del Museo Argentino de Ciencias Naturales "Bernardino Rivadavia", 13 (1): 59-68.

Zamorano, M. \& Brandoni, D. (2013). Phylogenetic analysis of the Panochthini (Xenarthra, Glyptodontidae), with remarks on their temporal distribution. Alcheringa 37:442-451. https://doi.org/10.1080/03115518. 2013.770224

Zamorano, M., Scillato-Yané, G.J. \& Zurita A.E. (2014). Revisión del género Panochthus (Xenarthra, Glyptodontidae). Revista Museo La Plata, SeccionPaleontologia, 14(72): 1-46.

Zamorano, M.; De los Reyes, M.; Poiré, D.G. \& Scillato-Yané, G.J. (2015). Primer registro fehaciente de Nopachtus coagmentatus (Xenarthra, Cingulata, Glyptodontidae) en la región Pampeana, Argentina. Contexto estratigráfico. Estudios Geológicos, 71 (1): 027.

Zamorano, M.; Toriño, P.; Rinderknecht, A. \& Perea, D.A. (2016). Primeros registros del género Nopachtus Ameghino (Xenarthra, Cingulata, Glyptodontidae) en Uruguay. Revista Mexicana de Biodiversidad 87(1): 62-67. http://doi. org/10.1016/j.rmb.2015.09.018

Zurita, A.E. (2007). Sistemática y evolución de los Hoplophorini (Xenarthra, Glyptodontidae, Hoplophorinae. Mioceno tardío-Holoceno temprano). Importancia bioestratigráfica, paleobiogeográfica y paleoambiental. Tesis doctoral, Universidad Nacional de la Plata, $367 \mathrm{pp}$. 